\title{
Statistical Modeling of Dual-Polarized MIMO Land Mobile Satellite Channels
}

\author{
Konstantinos P. Liolis, Student Member, IEEE, Jesús Gómez-Vilardebó, Student Member, IEEE, \\ Enrico Casini, Student Member, IEEE, and Ana I. Pérez-Neira, Senior Member, IEEE
}

\begin{abstract}
This Letter addresses the statistical modeling of dual-polarized MIMO-LMS fading channels. In the absence of accurate experimental results, a statistical model for the characterization of MIMO-LMS channels is proposed based on consolidation of available experimental results for SISO-LMS and MIMO wireless channels as well as on their extrapolation to the MIMO-LMS case of interest. Moreover, a step-by-step methodology for the simulation and time-series generation of the proposed MIMO-LMS channel model is provided, which is useful for the design and performance assessment of MIMOLMS transmission systems. The proposed model incorporates the effects of all relevant critical channel aspects in a flexible and fully-parameterized way.
\end{abstract}

Index Terms-Fading channels, land mobile satellite (LMS), multiple-input multiple-output (MIMO), polarization diversity.

\section{INTRODUCTION}

$\mathbf{T}$ HE appealing gains obtained by MIMO techniques in terrestrial networks [1] generate further interest in investigating the applicability of the same principle in satellite networks [2]. However, the fundamental differences between the terrestrial and satellite channels make such applicability a non trivial and non straightforward task and, thus, a rather challenging and hot research topic [3]. These differences are mainly related to the line-of-sight (LOS) operation of satellite links and to the absence of scatterers in the vicinity of the satellite, which eliminate multipath fading profiles over the space segment and lead to an inherent rank deficiency of the MIMO channel matrix. Further details on such key differences, which justify the increasing interest of the research community in MIMO satellite communications, can be found in [3]-[5].

A possible solution to form a MIMO matrix channel in a satellite environment is the polarization diversity [5]-[8]. The present work focuses on the modeling of dual-polarized

Paper approved by G. K. Karagiannidis, the Editor for Fading Channels and Diversity of the IEEE Communications Society. Manuscript received August 26, 2009; revised January 1, 2010 and April 14, 2010.

This work was supported in part by the ESA/ESTEC Contract AO/15146/06/NL/JD "MIMO Applicability to Satellite Networks". Part of this work was presented at the 27th AIAA Int. Commun. Satell. Systems Conf., ICSSC 2009, Edinburgh, UK, June 2009. The review of this paper was coordinated by Prof. G.K. Karagiannidis.

K. P. Liolis was with the European Space Agency, Research and Technology Centre (ESA/ESTEC), Noordwijk, The Netherlands. He is now with the School of Electrical and Computer Engineering, National Technical University of Athens (NTUA), Greece (e-mail: kliolis@mail.ntua.gr).

J. Gómez-Vilardebó is with the Centre Tecnològic de Telecomunicacions de Catalunya (CTTC), Barcelona, Spain.

E. Casini was with ESA/ESTEC, Noordwijk, The Netherlands. He is now with the NATO C3 Agency, NII Communications Infrastructure Services, The Hague, The Netherlands.

A.I. Pérez-Neira is with the Department of Signal Theory and Communications, Technical University of Catalonia (UPC), Barcelona, Spain

Digital Object Identifier 10.1109/TCOMM.2010.091710.090507
MIMO LMS channels and proposes a statistical model for their characterization. A useful methodology for simulation and time-series generation of such MIMO LMS channels is also provided, which facilitates the design and end-to-end performance assessment of MIMO LMS transmission systems. To this end, of particular interest is the potential evolution of conventional SISO-based DVB-SH systems [9] to MIMOenabled ones [2]. Useful numerical results on the outage capacity of dual-polarized MIMO LMS channels are provided investigating the effect of several critical parameters, such as the polarization and temporal correlation, LOS shadowing, cross-polar discrimination (XPD), cross-polar coupling (XPC), elevation angle and user environment.

On the related work, statistical models for narrowband and wideband dual-polarized MIMO LMS channels are presented in [6] and [7], respectively, based on an S-band field measurement campaign in a satellite emulated environment. Their main limitation is the low elevation angle assumed (in the order of $15^{\circ}$ ), which is not usually the case in practical MIMO LMS system configurations. In addition, a 3D physical MIMO channel model for synthetic satellite-to-indoor links is proposed in [5]. Its main limitations are that it requires experimental validation and that its time-series generation can only be obtained in terms of time-consuming and complex electromagnetic simulations. Moreover, a simplified statistical model for narrowband dual-polarized MIMO LMS channels is presented in [8], which is based on consolidation of measured data available in the literature. Although simple and easy-tosimulate, this model requires experimental validation and does not effectively incorporate crucial channel aspects, such as the LOS shadowing, the temporal correlation and the different states induced by the channel temporal variations. Furthermore, an analytical statistical channel model for narrowband MIMO LMS channels is presented in [10]. Its main limitations are that it requires experimental validation and that certain critical channel aspects, such as the temporal and polarization correlation at both transmit and receive sides, the XPD and the XPC, are not taken into account.

Thus, the main contributions of the present work to the relevant literature are twofold:

(i) A statistical model for dual-polarized MIMO LMS channels is proposed which incorporates all relevant critical channel aspects, such as the polarization and temporal correlation, LOS shadowing effect, antenna's XPD, environment's XPC, elevation angle and user environment, in a flexible and fullyparameterized way.

(ii) A practical step-by-step methodology for easy and fast computer simulation and time-series generation of dual- 


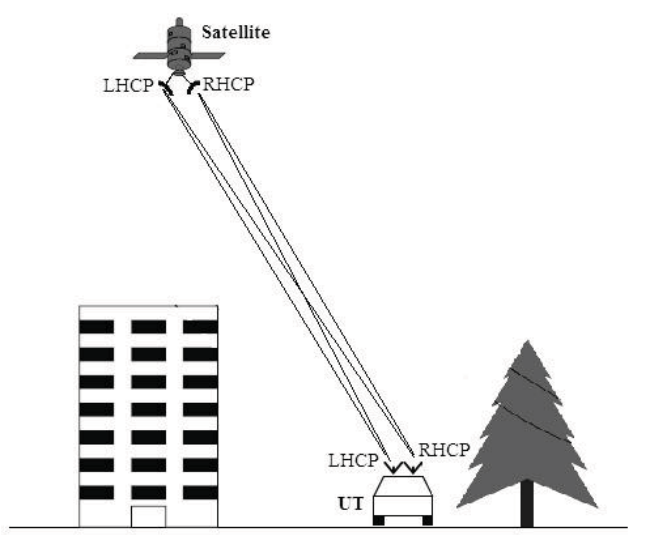

Fig. 1: 2x2 MIMO LMS channel: dual polarization diversity configuration.

polarized MIMO LMS channels is provided which is essentially needed in the design and end-to-end performance assessment of MIMO LMS transmission systems.

\section{Statistical MiMO-LMS Channel Modeling ANALYSIS}

Although there are no currently available MIMO LMS channel models which have been thoroughly validated against experimental data, statistical MIMO LMS channel models can be built up through careful consolidation of available experimental results and through their sound extrapolation to the MIMO LMS case of interest. In this regard, the present work takes into account the existing vast literature on SISO LMS and MIMO wireless channel modeling as well as some limited experimental results available in the MIMO LMS context as appropriate.

Fig. 1 illustrates the $2 \times 2$ MIMO LMS channel scenario under consideration. A single satellite employs a dual circularly polarized antenna with a right- and a left- hand circular polarized (R/L-HCP) element, whilst the mobile user terminal (UT) is also fitted with a similar dual circularly polarized antenna. Specifically, a single antenna capable of dual-polarization outputs (e.g., crossed dipoles to synthesize circular polarization with synthesized LHCP and RHCP outputs) is assumed, i.e., the multiple antenna elements are colocated on each side of the transmission link and there is no spatial separation assumed between them [11]. Regarding the operating frequency, satellite orbit and polarization employed, the baseline scenario in the DVB-SH context [9] is assumed, i.e., $\mathrm{S}(2 / 4 \mathrm{GHz})$ band, geostationary orbit (GEO) and circular polarization, respectively. The satellite elevation angle is denoted by $\theta$. Due to the local environment in the vicinity of the mobile UT, the satellite-UT LOS link might be clear, partially or fully obstructed which gives rise to multipath, shadowing and blockage effects. The resulting fading channel is assumed narrowband (i.e., frequency non-selective) since the multipath echoes are not significantly spread in time [12]-[14].

Under the assumptions above, the MIMO LMS channel assumed is modeled by a $2 \times 2$ MIMO channel matrix $\mathbf{H}=\left[h_{i j}\right]$ $(i, j=1,2) . h_{i j}$ represent the fading components of the SISO LMS sub-channels formed between the transmit and receive sides, which incorporate both the large-scale fading effects (i.e., those accounting for direct LOS shadowing) and the small-scale fading effects (i.e., those accounting for diffuse multipath). The critical channel aspects pertinent to the dual-polarized MIMO LMS channels under consideration are individually addressed and modeled next.

\section{A. SISO LMS Sub-Channels}

For the modeling of the envelope $|h i j|(i, j=1,2)$, the Loo (or else, shadowed-Ricean) distribution [12] is assumed because it has been fundamental to the building of the SISO LMS channel model in [13], which has been extensively used and validated in the recent standardization activity of DVBSH [9]. Under this assumption, the $2 \times 2$ MIMO LMS channel matrix $\mathbf{H}$ is expanded as

$$
\mathbf{H}=\left[h_{i j}\right]=\left[\bar{h}_{i j}\right]+\left[\tilde{h}_{i j}\right]=\overline{\mathbf{H}}+\tilde{\mathbf{H}} \quad(i, j=1,2)
$$

where

$$
\begin{aligned}
& h_{i j}=\left|h_{i j}\right| \exp \left(j \phi_{i j}\right) \\
& \quad=\left|\bar{h}_{i j}\right| \exp \left(j \bar{\phi}_{i j}\right)+\left|\tilde{h}_{i j}\right| \exp \left(j \tilde{\phi}_{i j}\right) \quad(i, j=1,2) \\
& p\left(\left|h_{i j}\right|\right)=\frac{\left|h_{i j}\right|}{b_{0} \sqrt{2 \pi d_{0}}} \times \\
& \int_{0}^{\infty} \frac{1}{z} \exp \left[-\frac{(\ln z-\mu)^{2}}{2 d_{0}}-\frac{\left|h_{i j}\right|^{2}+z^{2}}{2 b_{0}}\right] I_{0}\left(\frac{\left|h_{i j}\right| z}{b_{0}}\right) d z
\end{aligned}
$$

$\bar{\phi}_{i j}, \tilde{\phi}_{i j}$ are uniformly distributed over $[0,2 \pi], p($.$) is the$ Loo probability density function, $a=20 \log _{10}(\exp (\mu))$ and $\psi=20 \log _{10}\left(\exp \left(\sqrt{d_{0}}\right)\right)$ are the mean and standard deviation, respectively, of the lognormally distributed envelope $\left|\bar{h}_{i j}\right|$ of the large-scale fading components, $M P=10 \log _{10}\left(2 b_{0}\right)$ is the average power of the Rayleigh distributed envelope $\left|\tilde{h}_{i j}\right|$ of the small-scale fading components, and $I_{0}(\Delta)$ is the modified Bessel function of first kind and zero order.

The Loo statistical parameter triplet $(\alpha, \psi, M P)$ refers to the experimental dataset which is originally presented in [13] and further revised in [14]. Its specific choice is pertinent to each operational scenario assumed, i.e., to each frequency band: $\mathrm{L}(1 / 2 \mathrm{GHz})$ and $\mathrm{S}(2 / 4 \mathrm{GHz})$; to each user environment: intermediate/heavy/light tree shadowed, urban, suburban, open rural; and to each elevation angle: $\theta=40^{\circ}-80^{\circ}$ (S-band) and $\theta=10^{\circ}-70^{\circ}$ (L-band). The methodology to obtain the Loo statistical parameters $(\alpha, \psi, M P)$ based on tabulated experimental datasets is detailed in [14].

\section{B. Cross-Polarization Discrimination Effects}

The XPD of the large-scale fading components $\bar{h}_{i j}(i, j=$ $1,2)$ is related only to the cross-polar discrimination of the antenna, denoted by $X P D_{a n t}$, whereas the XPD of the small-scale fading components $\tilde{h}_{i j}(i, j=1,2)$ is related to both $X P D_{a n t}$ and the XPC of the propagation environment, denoted by $X P C_{e n v}$. Note that the antenna which is mainly critical in the presented analysis is that of the UT (i.e., reception side), whose XPD in most practical configurations of satellite networks is not greater than $15 \mathrm{~dB}$. On the contrary, the XPD of the satellite antenna (i.e., transmission side) is assumed to approximate $\infty$ due to its practically very large 
value. Thus, $X P D_{\text {ant }}$ will denote hereinafter only the UT antenna XPD, which critically affects the analysis.

The power imbalance between the co- and cross- polar components in the cases of $0^{\circ} / 90^{\circ}$ and $\pm 45^{\circ}$ polarization diversity configurations was investigated in detail in [15] and [16] in the context of fixed wireless and mobile radio channels, respectively, through experimental results and, in particular, through the Branch Power Ratio defined (in $\mathrm{dB}$ ) as

$$
B P R=10 \log _{10}\left(E\left[\left|h_{11}\right|^{2}\right] / E\left[\left|h_{22}\right|^{2}\right]\right)
$$

where $E[\cdot]$ denotes the expectation operator. In most examined scenarios, specifically, those related to the $\pm 45^{\circ}$ polarization diversity case, it was found that $\mathrm{BPR}=0 \mathrm{~dB}$, i.e., symmetry can be assumed. Thus, for the R/L-HCP circular polarization diversity of interest, symmetry is also assumed. This assumption is in line with experimental results obtained in the context of LMS channels and reported in [7], which further indicate such power balance. Based on this symmetry assumption, the power of the small- and large-scale fading components is given by

$$
\begin{gathered}
E\left[\left|\bar{h}_{\mathrm{ij}}\right|^{2}\right]=\left\{\begin{array}{cc}
\left(\psi^{2}+\alpha^{2}\right) \cdot\left(1-\beta_{\text {ant }}\right) & i=j \\
\left(\psi^{2}+\alpha^{2}\right) \cdot \beta_{\text {ant }} & i \neq j
\end{array}\right. \\
E\left[\left|\tilde{h}_{\mathrm{ij}}\right|^{2}\right]=\left\{\begin{array}{cc}
M P \cdot(1-\gamma) & i=j \\
M P \cdot \gamma & i \neq j
\end{array}\right.
\end{gathered}
$$

where $i, j=1,2,(\alpha, \psi, M P)$ are expressed in linear scale (i.e., not in $\mathrm{dB}$ ), $\beta_{\text {ant }} \in[0,1]$ depends only on $X P D_{\text {ant }}$ and $\gamma \in[0,1]$ depends on both $X P D_{a n t}$ and $X P C_{e n v}$.

Concerning the relationship between the XPD modeling factors $\beta_{a n t}$ and $\gamma$ in (5)-(6) as well as the actual measurable parameters $X P D_{\text {ant }}$ and $X P C_{\text {env }}$, one gets

$$
\begin{gathered}
X P D_{a n t}=10 \log _{10}\left(E\left[\left|\bar{h}_{i i}\right|^{2}\right] / E\left[\left|\bar{h}_{i j}\right|^{2}\right]\right) \\
=10 \log _{10}\left[\left(1-\beta_{\text {ant }}\right) / \beta_{\text {ant }}\right] \\
X P C_{e n v}=10 \log _{10}\left[\left(1-\gamma_{e n v}\right) / \gamma_{e n v}\right] \\
\gamma=\beta_{\text {ant }}\left(1-\gamma_{e n v}\right)+\left(1-\beta_{\text {ant }}\right) \gamma_{\text {env }}
\end{gathered}
$$

Several works such as [6]-[8], [17], [18] propose specific values for parameters $X P D_{a n t}, X P C_{e n v}$ based on extensive measurement campaigns, which are also taken into account in this work for the specific considered scenario.

\section{Polarization Correlation of Large-Scale Fading Compo- nents}

Due to the huge earth-space distance and the co-location of multiple antenna elements at the UT and the satellite, the largescale fading components $\bar{h}_{i j}(i, j=1,2)$ undergo a strong polarization correlation. If $\overline{\mathbf{C}}$ denotes the $4 x 4$ positive semidefinite Hermitian covariance matrix for the large-scale fading components, a $2 \times 2$ channel matrix $\overline{\mathbf{H}}_{w, \text { corr }}$ with polarization correlated identically distributed, circularly symmetric complex Gaussian elements of zero mean and unit variance is generated as [19], [20]

$$
\operatorname{vec}\left(\overline{\mathbf{H}}_{w, \operatorname{corr} r}\right)=\overline{\mathbf{C}}^{1 / 2} \cdot \operatorname{vec}\left(\overline{\mathbf{H}}_{w}\right)
$$

where vec(.) denotes the operator which stacks a matrix into a vector columnwise, and $\overline{\mathbf{H}}_{w}$ is the $2 \times 2$ channel matrix with polarization uncorrelated, identically distributed, circularly symmetric complex Gaussian elements of zero mean and unit variance. After appropriately incorporating the mean $\alpha$ and standard deviation $\psi$ (both expressed in $\mathrm{dB}$ ) in the Gaussian matrix $\overline{\mathbf{H}}_{w, c o r r}$ and then exponentiating in order to generate the lognormal channel matrix, the polarization correlated $2 \times 2$ MIMO channel matrix $\overline{\mathbf{H}}$ accounting for the large-scale fading components comes up in the form

$$
\operatorname{vec}(\overline{\mathbf{H}})=10^{\left[\operatorname{vec}\left(\overline{\mathbf{H}}_{w, c o r r}\right) \cdot(\psi / 20)+(\alpha / 20)\right]}
$$

Experimental results characterizing $\overline{\mathbf{C}}$ in the MIMO LMS context are reported in [6]. Although these results refer to low satellite elevation angles, the polarization correlation coefficients affecting the large-scale fading components get relatively high values (i.e., close to 1 ) for all user environments examined. This indicates that even in the more general and practical case of higher elevation angles, the polarization correlation coefficients will also get similarly high values and thus the same matrices $\overline{\mathbf{C}}$ are also considered in the present work (see Table I).

\section{Polarization Correlation of Small-Scale Fading Compo- nents}

Due to the angular spread of the multipath components and the co-location of multiple antenna elements at the UT and the satellite, the small-scale fading components $\tilde{h}_{i j}(i, j=1,2)$ also suffer from polarization correlation. If $\tilde{\mathbf{H}}_{w}$ is a $2 \times 2$ channel matrix with independent identically distributed zeromean circularly symmetric complex Gaussian elements of variance $M P$, and $\tilde{\mathbf{C}}$ is the $4 \times 4$ positive semi-definite Hermitian covariance matrix for the small-scale fading components, the polarization correlated small-scale fading component $\tilde{\mathbf{H}}$ is generated as [19], [20]

$$
\operatorname{vec}(\tilde{\mathbf{H}})=\tilde{\mathbf{C}}^{1 / 2} \cdot \operatorname{vec}\left(\tilde{\mathbf{H}}_{w}\right)
$$

where

$$
\tilde{\mathbf{C}}=\tilde{\mathbf{R}}_{t x}^{T} \otimes \tilde{\mathbf{R}}_{r x}
$$

In (13), $\otimes$ denotes the Kronecker product operator, the superscript $T$ denotes matrix transposition, and $\tilde{\mathbf{R}}_{t x}, \tilde{\mathbf{R}}_{r x}$ are the $2 \times 2$ positive semi-definite Hermitian, covariance matrices of the transmit and receive sides, respectively. Based on (12)-(13) and after employing straightforward algebra, the polarization correlated small-scale fading component $\tilde{\mathbf{H}}$ is given by [19], [20]

$$
\tilde{\mathbf{H}}=\tilde{\mathbf{R}}_{r x}^{1 / 2} \cdot \tilde{\mathbf{H}}_{w} \cdot \tilde{\mathbf{R}}_{t x}^{1 / 2}
$$

Moreover, based on (1), (6) and after employing straightforward algebra, the covariance matrices $\tilde{\mathbf{R}}_{t x}, \tilde{\mathbf{R}}_{r x}$ in (14) are given by

$$
\begin{aligned}
\tilde{\mathbf{R}}_{r x} & =E\left[\tilde{\mathbf{H}} \tilde{\mathbf{H}}^{H}\right] \\
& =M P \cdot\left[\begin{array}{cc}
1 & 2 \sqrt{(1-\gamma) \gamma} \tilde{\rho}_{r x} \\
2 \sqrt{(1-\gamma) \gamma} \tilde{\rho}_{r x} & 1
\end{array}\right] \\
\tilde{\mathbf{R}}_{t x} & =E\left[\tilde{\mathbf{H}}^{H} \tilde{\mathbf{H}}\right] \\
& =M P \cdot\left[\begin{array}{cc}
1 & 2 \sqrt{(1-\gamma) \gamma} \tilde{\rho}_{t x} \\
2 \sqrt{(1-\gamma) \gamma} \tilde{\rho}_{t x} & 1
\end{array}\right]
\end{aligned}
$$


where the superscript $H$ denotes matrix conjugate transposition and $\tilde{\rho}_{r x}, \tilde{\rho}_{t x}$ are the polarization correlation coefficients introduced in the small-scale fading components at the receive and transmit sides, respectively. Several works such as [6]-[8], [17] propose specific values for $\tilde{\rho}_{r x}, \tilde{\rho}_{t x}$ based on extensive measurement campaigns, which are also taken into account in this work for the specific considered scenario.

\section{E. Temporal Correlation}

To model the temporal correlation induced as the UT moves at a certain speed $v$ in the LMS environment, the following two different scales of channel temporal variations are considered:

(i) Inter-state temporal variations: In the SISO-LMS case, the temporal evolution of the large-scale fading components is characterized by a stochastic process $c(t)$ given by

$$
c(t)= \begin{cases}0 & B A D \\ 1 & G O O D\end{cases}
$$

which is modeled by a first-order 2-state Markov chain model with given absolute state probability matrix $\boldsymbol{W}_{\text {SISO }}$ and state transitions probability matrix $\boldsymbol{P}_{S I S O}$ for the specific scenario considered [14]. In the $2 \times 2$ MIMO LMS case, as described in Section II-C, the polarization correlation coefficient gets relatively high values, which further indicates that all the SISO LMS sub-links between the satellite and the UT follow the same sequences of "GOOD" and "BAD" channel states. Thus, the resulting Markov chain model for the MIMO LMS case of interest is also a 2-state one with absolute state and state transitions probability matrices being the same as in the SISO LMS case.

(ii) Intra-state temporal variations: Regarding the intrastate channel temporal variations, within each channel state, as the mobile UT moves at a certain speed v, the large-scale fading components undergo a temporal correlation. To model a specific coherence distance, there is a low-pass filtering process introduced in the large-scale fading components as detailed $[21]^{1}$. In this regard, for each state, a Gaussian random sequence of samples with zero mean and unit variance is first generated. One sample per transmission block of duration $T$ is assumed. These Gaussian samples are then low-pass filtered in order to introduce the temporal correlation, using the following low-pass IIR (Infinite Impulse Response) filter of one coefficient

$$
y_{n}=x_{n}+A \cdot y_{n-1}
$$

where $A=\exp \left(-v T / r_{c}\right), v$ is the speed of the mobile UT, $T$ is the sampling time being equal to the transmission block, and $r_{c}$ is the coherence distance. The filtered samples are then scaled by $\left(1-A^{2}\right)$ in order to restore the statistics of the samples before the filtering. Finally, based on (10)-(11), the uncorrelated Gaussian samples generated (incorporated in matrix $\overline{\mathbf{H}}_{w}$ ) are transformed to polarization correlated lognormal samples (incorporated in matrix $(\overline{\mathbf{H}})$ for a given covariance matrix $\overline{\mathbf{C}}$ and statistical parameters $(\alpha, \psi)$.

\footnotetext{
${ }^{1}$ Although originally developed for terrestrial mobile radio systems, the model in [21] has also been successfully validated through experimental results for LMS systems, as described in [6].
}

Regarding the small-scale fading components, to model their induced temporal correlation within each state, a simplified approach to decrease CPU simulation time is followed. To this end, the generated multipath channel samples are assumed constant during a transmission block $T$ (i.e., full temporal correlation within each block) but change independently from block to block. $T$ is assumed equal to the channel coherence time, i.e., the channel is sampled only once per block, which significantly reduces the CPU simulation time. Note that the assumed block fading model suffices to conduct statistical outage analysis [22] (e.g., outage capacity analysis in Section IV) in delay limited systems [23], as is the case in DVB-SH systems with relatively short FEC code [9] and where the LMS channel is slowly fading. In the more general case where this assumption of transmission block $\mathrm{T}$ does not hold, other more sophisticated approaches modeling the Doppler spectrum with a low-pass low-order Butterworth filter [14] can be followed, albeit at a cost of simulator complexity and CPU time increase. The incorporation of such Doppler spectrum models in the MIMO LMS channel simulator considered is left for future work.

\section{MiMO LMS Channel Simulator Methodology}

The following step-by-step procedure outlines a useful methodology for computer simulation and time-series generation of the presented dual-polarized MIMO LMS channels. The channel time-series generator can be easily integrated in a comprehensive end-to-end system simulator for detailed design and performance assessment of advanced MIMO LMS transmission systems [2].

\section{Step 1: Definition of Markov chain and generation of random walk}

- Obtain state frame length, absolute state probability matrix $\boldsymbol{W}_{M I M O}=\boldsymbol{W}_{S I S O}$ and state transition probability matrix $\boldsymbol{P}_{M I M O}=\boldsymbol{P}_{S I S O}$ for the 2-state Markov chain model from [14] for the specific user environment assumed.

- Let $P(i, j)$ denote the transition probability from state $i$ to state $j, S_{t}$ the current state and $S_{t+1}$ the next state. Given $S_{t}, S_{t+1}$ can be generated as follows: Generate $U$ Uniform $(0,1)$; Set $i=1$ and test the condition $U \leq \sum_{j=1}^{i} P\left(S_{t}, i\right)$; If true, the new state is $S_{t+1}=i$; Otherwise, test the same condition for $i=i+1$.

Step 2: Calculation of Loo statistical parameter set $(\alpha, \psi, M P)$

- Each time a new channel state is reached, a Loo statistical parameter triplet $(\alpha, \psi, M P)$ is drawn from the corresponding joint distribution and for the specific user environment assumed, as detailed in [14].

\section{Step 3: Generation of large-scale fading components}

- For each state and for each polarization dimension, a Gaussian random sequence of samples with zero mean and unit variance is generated. One sample per transmission block of duration $\mathrm{T}$ is assumed.

- These samples are then low-pass filtered in order to introduce the temporal correlation, using the one-coefficient low-pass IIR filter of (18). 

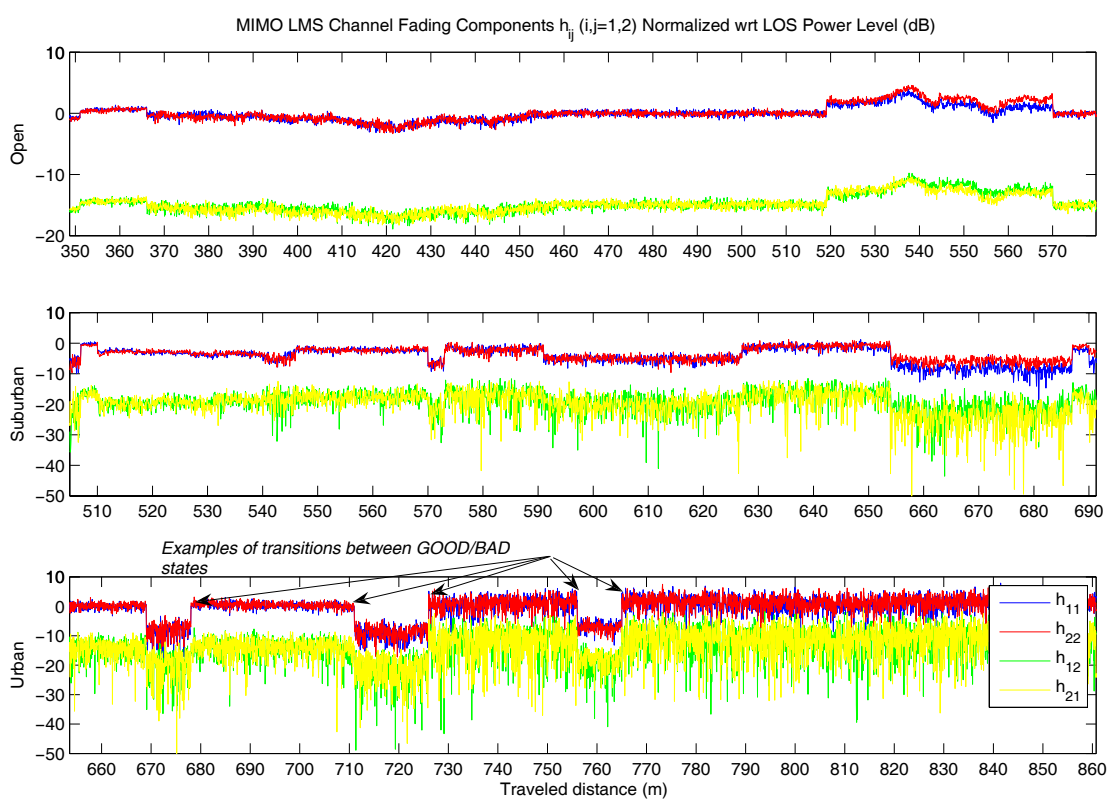

Fig. 2: Time series generated for $2 \times 2$ MIMO LMS channel in open rural, suburban and urban environment. MIMO LMS channel components $h_{i j}(i, j=1,2)$ normalized with respect to the LOS power level (in dB) vs. UT traveled distance. Input parameters assumed are reported in Table I.

- The filtered samples are then scaled by $\left(1-A^{2}\right)$ in order to restore the statistics of the samples before the filtering.

- The polarization correlation among the Gaussian generated samples of each dimension is introduced using the covariance matrix and the related operation in (10), which results in a Gaussian polarization correlated $2 \times 2$ matrix with zero mean and unit variance.

- According to the lognormal statistical parameters $(\alpha, \psi)$ drawn in Step 2, these Gaussian polarization correlated samples generated are then exponentiated accordingly as shown in (11) in order to generate the polarization correlated lognormally distributed $2 \times 2$ channel matrix $\overline{\mathbf{H}}$.

- The XPD effects are then introduced for the specific user environment assumed through the related operations in (5), which results in the channel matrix $\overline{\mathbf{H}}$ of (1).

\section{Step 4: Generation of small-scale fading components}

- Generate a $2 \times 2$ channel matrix $\tilde{\mathbf{H}}_{w}$ assuming independent identically distributed zero-mean circularly symmetric complex Gaussian elements with variance $M P$.

- Within each channel state, assume that the generated multipath channel samples are constant during a transmission block $\mathrm{T}$ but change independently from block to block. $T$ is assumed equal to the channel coherence time, i.e., the channel is sampled only once per block.

- The polarization correlation among elements of $\tilde{\mathbf{H}}_{w}$ is introduced using the multipath covariance matrices $\tilde{\mathbf{R}}_{t x}$, $\tilde{\mathbf{R}}_{r x}$ through the operations in (14), which results in the polarization correlated small-scale fading matrix $\tilde{\mathbf{H}}$.

- The XPD effects are then introduced for the specific user environment assumed through the related operations in (6), which results in the channel matrix $\tilde{\mathbf{H}}$ of (1).

Step 5: Combination of large- and small-scale fading components
- Combine the generated large- and small-scale fading components by simply adding the signals in each MIMO channel at each time sample as indicated in (1), that is, $\mathbf{H}=\overline{\mathbf{H}}+\tilde{\mathbf{H}}$. Note that the produced time series are normalized with respect to the LOS power level since the Loo statistical parameters $(\alpha, \psi, M P)$ in [14] are normalized, as well.

\section{NumericAl Results ANd Discussion}

Next, useful numerical results are provided based on the proposed MIMO LMS channel model. To this end, specific MIMO LMS channel scenarios are assumed whose consolidated parameters pertinent to the analysis are given in Table I. Unless otherwise stated below, the rest of the relevant parameters assumed for Fig. 2-5 are those reported in Table I.

First, the time series generated for the $2 \times 2$ MIMO LMS channel assuming operation in an open rural, suburban and urban environment is illustrated in Fig. 2. A sequence of "GOOD" and "BAD" channel states along the UT traveled distance can be seen. Within each user environment, note that the level of all "GOOD" states is not the same. This applies also for all "BAD" states and it is because every time a new channel state is reached along the UT traveled distance, a new Loo statistical parameter triplet $(\alpha, \psi, M P)$ is drawn from the corresponding joint distribution. The increase in the amount of multipath fading when moving from an open rural to an urban environment can also be observed. Moreover, it can be seen that the channel XPD between the co-polar $h_{i i}(i=j=1,2)$ and the cross-polar $h_{i j}(i \neq j=1,2)$ components is not constant along the UT traveled distance, which is mainly due to the $X P C_{e n v}$. The temporal and polarization correlation between each of the components of the channel samples is also effectively incorporated. 


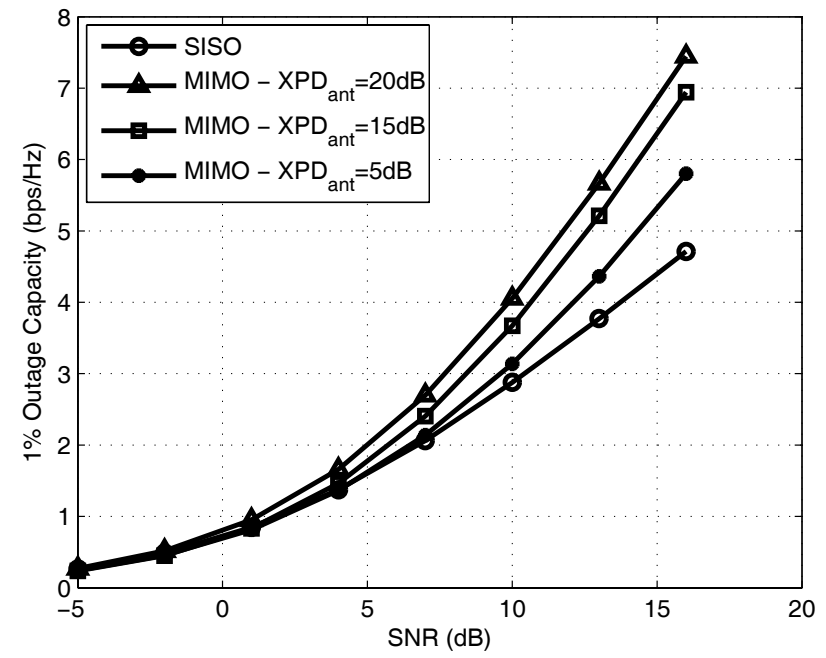

Fig. 3: $1 \%$ outage capacity of $2 \times 2$ MIMO LMS channel vs. SNR in open rural environment. Effect of antenna crosspolarization discrimination, $X P D_{\text {ant }}$.

In Fig. 3-5 below, the $1 \%$ outage capacity achieved is evaluated through Monte Carlo simulations over $10^{4}$ channel realizations using the proposed MIMO LMS channel simulator. The respective SISO cases are also plotted for the sake of comparison as appropriate. The outage capacity calculated is defined in [1]. Specifically, in Fig. 3, the $1 \%$ outage capacity of a 2x2 MIMO LMS channel assuming operation in an open rural environment is plotted vs. SNR in order to investigate the effect of the terminal antenna XPD. As $X P D_{\text {ant }}$ increases, the cross-polar interferences become weaker, the MIMO channel becomes diagonal and the outage capacity achieved increases. Moreover, in Fig. 4, the $1 \%$ outage capacity of a 2x2 MIMO LMS channel assuming operation in a suburban environment is plotted vs. SNR in order to investigate the effect of the satellite elevation angle. As $\theta$ decreases, the signal is blocked with higher probability, the "BAD" state becomes more dominant and thus the outage capacity achieved by the respective MIMO case decreases. In addition, in Fig. 5, the 1\% outage capacity of a 2x2 MIMO LMS channel assuming operation in an urban environment is plotted vs. SNR in order to investigate the effect of the polarization correlation between the small-scale fading components. As $\tilde{\rho}_{r x}$ (or $\tilde{\rho}_{t x}$ ) decreases, the outage capacity achieved by each considered MIMO case increases. With respect to Fig. 3-4, Fig. 5 indicates that the outage capacity achieved in an urban environment is much lower than that in open and suburban environments. However, in all cases simulated above and depicted in Fig. 3-5, including the case of the harsh urban environment where the satellite signal is usually blocked, there is a significant capacity gain evident between the respective MIMO LMS and SISO LMS performance curves.

\section{CONCLUSIONS}

In this letter, a novel statistical channel model for the characterization of dual-polarized MIMO LMS channels is presented along with a step-by-step methodology for their

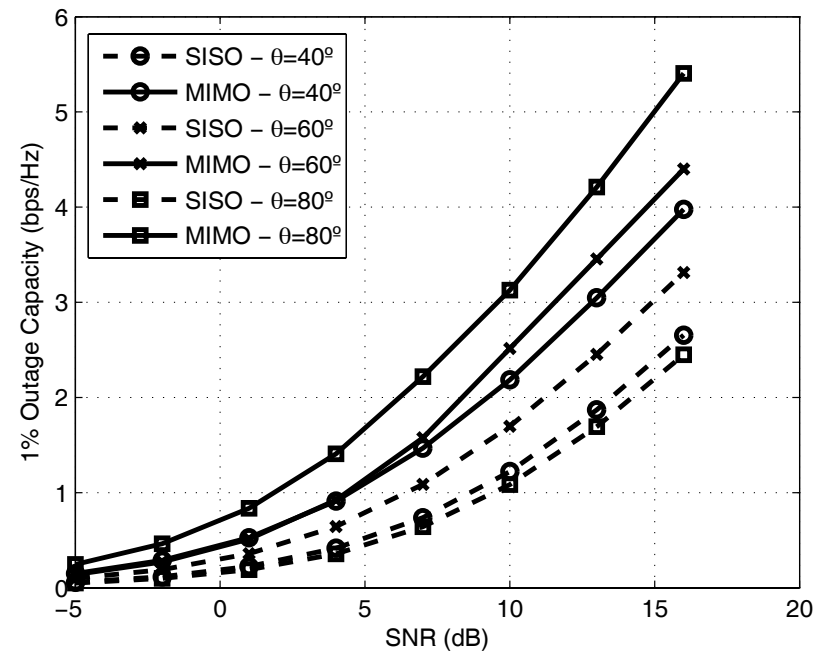

Fig. 4: $1 \%$ outage capacity of $2 \times 2$ MIMO LMS channel vs. SNR in suburban environment. Effect of satellite elevation angle, $\theta$.

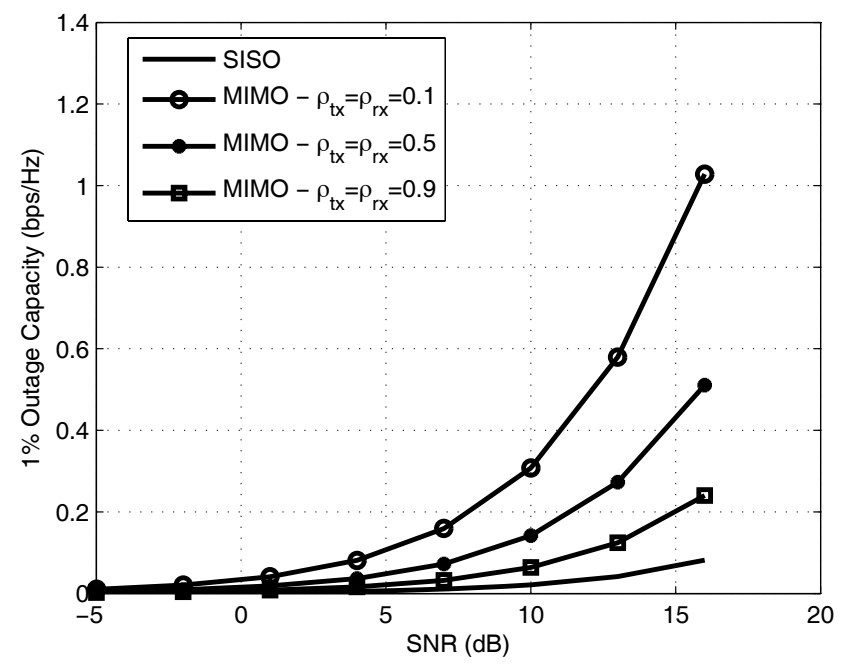

Fig. 5: $1 \%$ outage capacity of $2 \times 2$ MIMO LMS channel vs. SNR in urban environment. Effect of polarization correlation of small-scale fading components, $\tilde{\rho}_{r x}, \tilde{\rho}_{t x}$. The special case of $\tilde{\rho}_{r x}=\tilde{\rho}_{t x}$ is assumed and only the "BAD" state is considered, as more relevant in urban environments.

time-series generation and computer simulation. The proposed channel model requires further experimental validation but it is versatile, flexible, fully parameterized and incorporates the effects of all relevant critical channel aspects. Thus, the values considered for its various modeling parameters can be further fine-tuned upon availability of new experimental results. Moreover, the proposed channel simulator methodology is useful and allows detailed design and end-to-end performance assessment of advanced MIMO LMS transmission systems. Outage capacity statistics are also provided based on which the effect of several critical channel modeling parameters, such as the polarization and temporal correlation, LOS shadowing, XPD, XPC, elevation angle and user environment, are investigated. In all simulated cases, the observed capacity gains 
TABLE I: Parameters Assumed for Dual-Polarized MIMO LMS Channel Scenarios

\begin{tabular}{|c|c|c|c|c|}
\hline Parameter & Open Rural Environment & Suburban Environment & Urban Environment & Reference \\
\hline Operating frequency, $f$ & $2.2 \mathrm{GHz}$ ( $\mathrm{S}$ band) & $2.2 \mathrm{GHz}$ ( $\mathrm{S}$ band) & $2.2 \mathrm{GHz}$ (S band) & [9] \\
\hline Satellite orbit & GEO & GEO & GEO & [9] \\
\hline Polarization & RHCP \& LHCP & RHCP \& LHCP & RHCP \& LHCP & [9] \\
\hline Mobile UT speed, $v$ & $50 \mathrm{~km} / \mathrm{h}$ & $50 \mathrm{~km} / \mathrm{h}$ & $50 \mathrm{~km} / \mathrm{h}$ & [9] \\
\hline Satellite elevation angle, $\theta$ & $40^{\circ}$ & $40^{\circ}$ & $40^{\circ}$ & [9] \\
\hline XPD of environment, $X P C_{e n v}$ & $15 \mathrm{~dB}$ & $6 \mathrm{~dB}$ & $5 \mathrm{~dB}$ & [8], [17] \\
\hline XPD of UT antenna, $X P D_{a n t}$ & $15 \mathrm{~dB}$ & $15 \mathrm{~dB}$ & $15 \mathrm{~dB}$ & {$[8],[17]$} \\
\hline $\begin{array}{l}\text { Loo statistical parameter } \\
\text { triplet }(\alpha, \psi, M P)\end{array}$ & \multicolumn{3}{|c|}{$\begin{array}{l}\text { For each environment, each time a new state is reached, }(\alpha, \psi, M P) \\
\text { are drawn from corresponding joint distribution }\end{array}$} & [14] \\
\hline $\begin{array}{l}\text { Polarization correlation coefficient } \\
\text { of small-scale fading } \\
\text { components at Tx, } \tilde{\rho}_{t x}\end{array}$ & 0.4 & 0.5 & 0.5 & {$[8],[17]$} \\
\hline $\begin{array}{l}\text { Polarization correlation coefficient } \\
\text { of small-scale fading } \\
\text { components at Tx, } \tilde{\rho}_{r x}\end{array}$ & 0.5 & 0.5 & 0.5 & [8], [17] \\
\hline $\begin{array}{l}\text { Polarization correlation matrix of } \\
\text { large-scale fading components, } \\
\qquad \overline{\mathbf{C}}\end{array}$ & 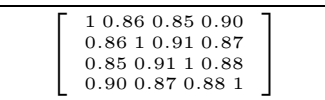 & $\begin{array}{l}10.76 \quad 0.76 \quad 0.83 \\
0.76 \quad 10.830 .75 \\
0.760 .8310 .78 \\
0.830 .750 .781\end{array}$ & $\begin{array}{llll}1 & 0.86 & 0.86 & 0.92 \\
0.86 & 0.89 & 0.85 \\
0.86 & 0.89 & 0.89 \\
0.92 & 0.85 & 0.93 & 1\end{array}$ & [6] \\
\hline Inter-state temporal variations & $\begin{array}{l}\text { For each environment, fir } \\
\text { and state transitions pro }\end{array}$ & $\begin{array}{l}\text { der 2-state Markov chain } \\
\text { lity matrices } \mathbf{W}_{M I M O}=\end{array}$ & $\begin{array}{l}\text { h respective absolute state } \\
\text { and } \mathbf{P}_{M I M O}=\mathbf{P}_{S I S O}\end{array}$ & [14] \\
\hline
\end{tabular}

between the respective MIMO LMS and SISO LMS channels are considerable.

\section{ACKNOWLEDGMENTS}

The authors would like to thank Dr. R. De Gaudenzi from ESA/ESTEC for the fruitful discussions on MIMO LMS systems as well as Mr. R. Prieto-Cerdeira from ESA/ESTEC for the relevant material made available on the revised SISO LMS channel model. They would also like to thank Dr. P.-D. M. Arapoglou from NTUA for his detailed review and comments. The authors would like also to thank the Associate Editor and the three anonymous reviewers whose useful comments helped to improve the original version of this article.

\section{REFERENCES}

[1] A. J. Paularaj, R. Nabar, D. Gore, and H. Bolcskei, "An overview of MIMO communications-a key to gigabit wireless," Proc. IEEE, vol. 92, no. 2, pp. 198-218, Feb. 2004.

[2] A. I. Pérez-Neira, C. Ibars, J. Serra, A. del Coso, J. Gómez, and M. Caus, "MIMO applicability to satellite networks," in Proc. Int. Work. Signal Process. Space Commun. SPSC, Rhodes, Greece, Oct. 2008.

[3] P.-D. M. Arapoglou, K. P. Liolis, M. Bertinelli, A. D. Panagopoulos, P. G. Cottis, and R. De. Gaudenzi, "MIMO over satellite: a review," IEEE Commun. Surveys Tutorials, $1^{\text {st }}$ Quarter, 2011.

[4] K. Liolis, A. Panagopoulos, and P. Cottis, "Multi-satellite MIMO communications at KU band and above: investigations on spatial multiplexing for capacity improvement and selection diversity for interference mitigation," EURASIP J. Wireless Commun. Netw., June 2007.

[5] P. Horvath, G. K. Karagiannidis, P. R. King, S. Stavrou, and I. Frigyes, "Investigations in satellite MIMO channel modeling: accent on polarization," EURASIP J. Wireless Commun. Netw., June 2007.

[6] P. King, "Modeling and measurement of the land mobile satellite MIMO radio propagation channel," Ph.D. dissertation, University of Surrey, UK, June 2007.

[7] P. King and S. Stavrou, "Low elevation wideband land mobile satellite MIMO channel characteristics," IEEE Trans. Wireless Commun., vol. 6, no. 7, pp. 2712-2720, July 2007.

[8] M. Sellathurai, P. Guinand, and J. Lodge, "Space-time coding in mobile satellite communications using dual-polarized channels," IEEE Trans. Veh. Technol., vol. 55, no. 2, Jan. 2006.
[9] ETSI TS 102584 V1.1.1 (2008-12), Digital Video Broadcasting (DVB) Guidelines for Implementation for Satellite Services to Handheld devices $(\mathrm{SH})$ below $3 \mathrm{GHz}$.

[10] G. Alfano and A. D. Maio, "A theoretical framework for LMS MIMO communicationsystems performance analysis," in Proc. 3rd Int. Waveform Diversity Design Conf., June 2007.

[11] C. Oestges, B. Clerckx, M. Guillaud, and M. Debbah, "Dual-polarized wireless communications: from propagation models to system performance evaluation," IEEE Trans. Wireless Commun., vol. 7, no. 10, pp. 4019-4031, Oct. 2008.

[12] C. Loo, "A statistical model for a land mobile satellite link," IEEE Trans. Veh. Technol., vol. 34, no. 3, pp. 122-127, Aug. 1985.

[13] F. P. Fontán, M.-A. V. Castro, C. E. Cabado, J. P. García, and E. Kubista, "Statistical modeling of the LMS channel," IEEE Trans. Veh. Technol., vol. 50, no. 6, pp. 1549-1567, Nov. 2001.

[14] R. P. Cerdeira, F. Pérez Fontán, P. Burzigotti, A. Bolea Alamañac, and I. Sanchez Lago, "Versatile two-state land mobile satellite channel model with first application to DVB-SH analysis," Int. J. Satell. Commun. Netw., DOI: 10.1002/sat.964, 2010.

[15] C. Oestges, V. Erceg, and A. Paulraj, "Propagation modeling MIMO multipolarized fixed wireless channels," IEEE Trans. Veh. Technol., vol. 53, no. 3, pp. 644-654, May 2004.

[16] T. B. Sørensen, A. Nielsen, P. E. Mogensen, M. Tolstrup, and K. Steffensen, "Performance of two-branch polarization antenna diversity in an operational GSM network," in IEEE VTC, Ottawa, Canada, May 1998.

[17] J. Lempiäinen and J. Laiho-Steffens, "The performance of polarization diversity schemes at a base station in small/micro cell at $1800 \mathrm{MHz}$," IEEE Trans. Veh. Technol., vol. 47, no. 3, Aug. 1998.

[18] T. Brown, S. Saunders, S. Stavrou, and M. Fiacco, "Characterization of polarization diversity at the mobile," IEEE Trans. Veh. Technol., vol. 56, no. 5, Sep. 2007.

[19] D.-S. Shiu, G. Foschini, M. Gans, and J. Kahn, "Fading correlation and its effect on the capacity of multielement antenna systems," IEEE Trans. Commun., vol. 48, no. 3, pp. 502-513, Mar. 2000.

[20] C.-N. Chuah, D. Tse, J. Kahn, and R. Valenzuela, "Capacity scaling in MIMO wireless systems under correlated fading," IEEE Trans. Inf. Theory, vol. 48, no. 3, pp. 637-650, Mar. 2002.

[21] M. Gudmundson, "Correlation model for shadow fading in mobile radio systems," IEE Electron. Lett., vol. 27, no. 23, pp. 2145-2146, 1991.

[22] L. Ozarow, S. Shamai, and A. Wyner, "Information theoretic considerations for cellular mobile radio," IEEE Trans. Veh. Technol., vol. 43, no. 2, pp. 359-378, May 1994.

[23] S. V. Hanly and D. N. Tse, "The multi-access fading channel: Shannon and delay limited capacities," in Proc. 33rd Allerton Conf., Monticello, IL, 1995. 